\title{
Effect of Electroacupuncture On Discomfort During Gastroscopy Without General Anesthetic: A Study Protocol For A Randomized Controlled Trial
}

\section{Binyu Yu}

Shanghai University of Traditional Chinese Medicine

Philippa Jemma Hazlewood

Shanghai University of Traditional Chinese Medicine

\section{Xuan Yin}

Shanghai University of Traditional Chinese Medicine

Shanshan Li

Shanghai University of Traditional Chinese Medicine

\section{Hongyu Yue}

Shanghai University of Traditional Chinese Medicine

\section{Kun Xu}

Shanghai University of Traditional Chinese Medicine

\section{Shifen Xu ( $\nabla$ xu_doctor@hotmail.com )}

Shanghai Municipal Hospital of Traditonal Chinese Medicine, Shanghai University of Traditional

Chinese Medicine https://orcid.org/0000-0001-5595-3480

\section{Yiqun Mi}

Shanghai University of Traditional Chinese Medicine

\section{Study protocol}

Keywords: Electroacupuncture, gastroscopy, discomfort, randomized controlled trial

Posted Date: June 28th, 2021

DOl: https://doi.org/10.21203/rs.3.rs-502017/v1

License: (c) (1) This work is licensed under a Creative Commons Attribution 4.0 International License. Read Full License 


\section{Abstract}

Background: Gastroscopy procedures are frequently performed under general anesthetic to minimize discomfort. Patients who refuse a sedative injection may experience more discomfort and adverse reactions such as pain and nausea. These instances reduce patient compliance and willingness to participate in future procedures. Acupuncture has been shown to have an antinausea and analgesic effect, however there is limited data available that demonstrates acupuncture as effective when applied before gastroscopy.

Methods: A total of 60 participants will be randomly assigned to the electroacupuncture (EA) group and the sham electroacupuncture (SEA) group at a ratio of 1: 1. Acupuncture treatment will be performed before gastroscopy for a duration of 30 minutes. All patients will complete detailed questionnaires at 30 minutes and 7 days post-procedure to record the severity of their symptoms. The primary outcome will be the average of 4 standard visual analogue scales (VAS) scores in the categories of nausea,vomitting, throat discomfort and agitation as reported by the patient. The secondary outcomes will be patient's anxiety as recorded by the 6 Item Short Form of the State-Trait Anxiety Inventory (STAI-S6) and Amsterdam Pre-Operative Anxiety and Information Scale (APAIS), preference in a future endoscopy, oxygen saturation ( $\mathrm{SaO} 2)$, heart rate (HR) and blood pressure (BP). Anxiety scales will be assessed before and after acupuncture, others will be complete at 30 minutes and 7 days post-procedure.

Discussion: This randomized controlled trial will explore the feasibility of the further clinical application of electroacupuncture for the improvement of patient discomfort during gastroscopy without systemic sedation.

Trial registration: This trial has been approved by the Ethics Committee of Shanghai Municipal Hospital of Traditional Chinese Medicine (2020SHL-KY-11) and is registered with ChiCTR2000040726.

\section{Introduction}

\section{Background and rationale $\{6 a\}$}

Upper gastrointestinal endoscopy (gastroscopy) is one of the most common diagnostic and therapeutic methods of assessing upper gastrointestinal diseases. ${ }^{1}$ Gastroscopy is the first choice for clinical examination of upper gastrointestinal diseases because physicians can directly observe the inspection site and perform pathological biopsy and ligation on the diseased tissue in real time. ${ }^{2}$ Due to the aging population and modern dietary changes the average number of gastroscopies performed annually has sharply increased over the past few decades. ${ }^{3}$

The strong stimulation from gastroscope insertion can often induce a gag reflex, which causes throat discomfort and nausea. Unmedicated patients also experience anxiety, increased blood pressure and tachycardia during gastroscopy procedure. To curtail these symptoms, $98 \%$ of gastroscopies in the United States are performed with general anesthetic. However, the use of anesthesia is costly and 
associated with inhibited cognitive function and mental decline. Moreover, the post procedural impairment affects a patients' ability to drive and return to work immediately afterwards. ${ }^{4}$ That is why approximately half of Chinese gastroscopy patients decline general anesthesia during gastroscopy. 5,6 We therefore need to identify a safe and effective method to reduce adverse events during gastroscopy and offer patients a palatable alternative to general sedation. Acupuncture analgesia has its unique advantages. Clinical trials have confirmed that preoperative acupuncture can reduce anxiety, decrease the use of anesthetic drugs, relieve nausea and vomiting and shorten hospital stays. ${ }^{5-8}$ Acupuncture during the perioperative period can help restore intestinal function and reduce postoperative pain. ${ }^{9,10}$ One study has reported acupuncture analgesia as a successful alternative to pharmacological sedation, however this trial only used a small sample size and is quite dated. ${ }^{11}$ Therefore, high-quality randomized clinical trials are needed to re-validate previous findings and develop a clinical trial of electroacupuncture to reduce patients' discomfort during standard gastroscopy.

\section{Objectives $\{7\}$}

we designed a rigorous randomized controlled clinical trial using a single-center, single-blind research method to evaluate the impact of electroacupuncture (EA) on the discomfort of patients undergoing gastroscopy. We aim to observe: (1) whether EA can reduce the discomfort of patients during gastroscopy; (2) whether EA can improve preoperative anxiety and benefit patient's mid-operation blood pressure. The results will help prove whether EA is an effective and safe therapy to reduce patient discomfort during general gastroscopy.

\section{Trial design \{8\}}

This RCT is a single-site patient- and assessor-blinded placebo-controlled trial. It will be implemented in Shanghai Municipal Hospital of Traditional Chinese Medicine. All eligible patients will be randomly assigned to the electroacupuncture group(EA)or the sham electroacupuncture (SEA) group in a 1:1 allocation ratio. All patients must sign the informed consent form before they are enrolled in the trial. The research process flow chart is detailed in Fig. 1. Throughout the trial, the Consolidated Standards of Reporting Trials (CONSORT) $)^{12}$ and acupuncture reporting guidelines, Standards for Reporting Interventions in Clinical Trials of Acupuncture (STRICTA) ${ }^{13}$ were followed.

\section{Methods: Participants, Interventions And Outcomes Study setting $\{9\}$}

All eligible participants will be recruited through hospital-based advertisements within the outpatient clinic and on the Shanghai Municipal Hospital of TCM website.

\section{Eligibility criteria $\{10\}$}


Inclusion criteria

Patients who meet the following conditions will be included:

(1) 18-70 years old, male or female;

(2) Patients who will undergo gastroscopy with local anesthesia;

(3) Clear consciousness, can answer the questions, understand the scales and complete the assessment;

(4) Agree to participate and sign a written informed consent.

Exclusion criteria

Patients who meet any of the following conditions will be excluded:

(1) Patients with mental disorders or severe cognitive impairments who cannot participate in cooperation;

(2) Patients with a history of previous bleeding disorders, current use of warfarin or low-molecular-weight heparin;

(3) Patients who cannot receive acupuncture for reasons such as infections around the acupuncture points or allergy to acupuncture needles;

(4) Patients who have received acupuncture treatment in the past 6 months;

(5) Participants in other clinical trials that may interfere with the primary endpoint.

\section{Who will take informed consent? \{26a\}}

All participants will be recruited by advertising through Website of Shanghai Municipal Hospital of TCM. People who need gastroscopy and interested in participating can contact the researcher by calling or adding the Wechat. We will evaluate according to the inclusion and exclusion criteria. All participants will be clearly informed and understand about the potential benefits and risks of the trial before they sign the informed consent.
Additional consent provisions for collection and use of participant data and biological specimens $\{26 \mathrm{~b}\}$

Not applicable.

\section{Interventions}

\section{Explanation for the choice of comparators $\{6 \mathrm{~b}\}$}


In this trial, All participants will receive the electroacupuncture or sham electroacupuncture before the gastroscopy. We adopt the superficial acupuncture in the non-acupoints method, it has the advantages of good masking effect, not easily perceived by the patients, and with higher feasibility of operation.

\section{Intervention description $\{11$ a $\}$}

All participants will receive the electroacupuncture or sham electroacupuncture before the gastroscopy, then, they will undergo standard upper gastrointestinal endoscopy procedures. Patients in both groups will be discharged within 1 hour after the endoscopy, which includes a 30-minute observation period, a preference for future endoscopy assessment and blinding assessment. The acupuncture methods are described in Table 1.To maintain blinding. Patients will be asked to wear an eye-patch and receive treatment in a supine position in an isolated space with limited communication with the acupuncturist. The acupuncturist will disinfect the patient's skin with $75 \%$ alcohol cotton balls before treatment. Acupuncture treatment will last 30 minutes. After removing the needle, researchers will use a clean cotton ball to compress the acupoint to prevent bleeding. A total of 8 points will be used. Table 2 summarizes the acupoints and rationale.

Table 1

Treatment methods of electroacupuncture and acupoints

\begin{tabular}{|c|c|c|}
\hline & EA group & SEA group \\
\hline Acupoints & $\begin{array}{l}\text { Hegu(LI4), Neiguan(PC6), } \\
\text { Zusanli(ST36),Liangqiu(ST34) }\end{array}$ & Non-acupoints as shown in the Table 3 \\
\hline $\begin{array}{l}\text { Depth of } \\
\text { insertion }\end{array}$ & 10 mm:LI4 PC6;30mm:ST36 ST34 & Superficial acupuncture \\
\hline $\begin{array}{l}\text { Needle } \\
\text { type }\end{array}$ & $\begin{array}{l}\text { Steel needles (Wuxi Jiajian Medical Co. } \\
\text { Ltd. Wuxi, China) }\end{array}$ & The same as EA group \\
\hline $\begin{array}{l}\text { Needle } \\
\text { sensation }\end{array}$ & With de-qi sensation & Without de-qi sensation \\
\hline \multirow{4}{*}{$\begin{array}{l}\text { Electric } \\
\text { stimulation }\end{array}$} & Two pairs of needles: & Two pairs of needles: \\
\hline & LI4-ST36 (bilaterally) & LI4-ST36 (bilaterally) \\
\hline & $\begin{array}{l}\text { Connected to CMNS6-1(Wuxi Jiajian } \\
\text { Medical Device CO., China) }\end{array}$ & $\begin{array}{l}\text { Connected to CMNS6-1(Wuxi Jiajian } \\
\text { Medical Device CO., China) }\end{array}$ \\
\hline & $\begin{array}{l}\text { Deliver continuous wave-type low- } \\
\text { frequency } 2 \mathrm{HZ} \text { and current of } 2 \mathrm{~mA}\end{array}$ & No electrical current delivered \\
\hline EA electroac & uncture, SEA sham electroacupuncture & \\
\hline
\end{tabular}


Table 2

Acupoint selection and rationale based on traditional Chinese medicine

\begin{tabular}{|c|c|c|c|}
\hline Acupoint & Location & $\begin{array}{l}\text { Traditional } \\
\text { Chinese } \\
\text { medicine } \\
\text { indication }\end{array}$ & $\begin{array}{l}\text { Suggested } \\
\text { technique }\end{array}$ \\
\hline LI4(hegu) & $\begin{array}{l}\text { Dorsum of hand, at the level of the midpoint } \\
\text { of the second metacarpal bone, between the } \\
\text { first and second metacarpal bones }\end{array}$ & $\begin{array}{l}\text { Relieve } \\
\text { visceral pain }\end{array}$ & $\begin{array}{l}\text { Needle } \\
\text { perpendicular,0.5- } \\
1.0 \text { cun }\end{array}$ \\
\hline PC6(neiguan) & $\begin{array}{l}\text { Palmar aspect of the forearm, between the } \\
\text { tendons, } 2 \text { cun away from the transverse } \\
\text { crease of the wrist }\end{array}$ & $\begin{array}{l}\text { Reduce } \\
\text { nausea and } \\
\text { stomach } \\
\text { pain }\end{array}$ & $\begin{array}{l}\text { Needle } \\
\text { perpendicular, } 0.5- \\
1.5 \text { cun }\end{array}$ \\
\hline ST36(zusanli ) & $\begin{array}{l}\text { Antero-lateral leg, } 1 \text { middle-finger breadth } \\
\text { next to the anterior crestof tibia, } 3 \text { cun under } \\
\text { the depression lateral to the patellar } \\
\text { ligament }\end{array}$ & $\begin{array}{l}\text { Reduce } \\
\text { nausea and } \\
\text { stomach } \\
\text { pain }\end{array}$ & $\begin{array}{l}\text { Needle } \\
\text { perpendicular,1-2 } \\
\text { cun }\end{array}$ \\
\hline ST34(liangqiu) & $\begin{array}{l}2 \text { cun above the bottom of the patella, on the } \\
\text { line between the anterior superior iliac spine } \\
\text { and the lateral end of the bottom of the } \\
\text { patella }\end{array}$ & $\begin{array}{l}\text { Relieve } \\
\text { stomach } \\
\text { pain }\end{array}$ & $\begin{array}{l}\text { Needle } \\
\text { perpendicular, } 1- \\
1.2 \text { cun }\end{array}$ \\
\hline
\end{tabular}

- The electroacupuncture group

Participants in the treatment group will receive EA treatment. The standard acupuncture method will be applied at 8 acupoints: bilateral Hegu (LI4), Neiguan (PC6), Zusanli (ST36) and Liangqiu (ST34). All acupoints were selected based on literature, clinical experience and acupuncture textbooks. The acupuncture needles will be disposable sterile needles made of stainless steel $(0.25 \times 40 \mathrm{~mm}$ and $0.30 \times$ $40 \mathrm{~mm}$; Jia Jian, China). After insertion, needles will be manipulated using the lifting-thrusting and twirling technique. An electroacupuncture apparatus (CMNS6-1, Wuxi Jiajian Medical Device CO., China) will be connected to 2 pairs of needles (LI4, ST36 bilaterally) to deliver a continuous wave, low-frequency $(2 \mathrm{~Hz})$ and current of $2 \mathrm{~mA}$.

\section{- The Sham Electroacupuncture Group}

In the control group, we will use the same acupuncture needles as used in the EA group but repurposed as placebo needles. The acupuncturist will perform superficial acupuncture on the non-acupoints detailed in Table 3,and Picture1-4 show the location. All of the needles will be inserted using a needle tube to a superficial depth (2-3 $\mathrm{mm}$ ) without invoking the sensation of "De-qi". The electroacupuncture apparatus will be connected to 2 pairs of needles (LI4, ST36 bilaterally) for 30 min, but all indicators will be set to "0" to ensure no electrical current is delivered. 
Table 3 Non-acupoints positioning

Acupoints Non-acupoints

Hegu(LI4) 1 cun beside Zhigou(SJ6), between the sanjiao channel and the large intestine channel

Neiguan (PC6) In the middle of the medial epicondyle of the humerus and the styloid process of ulna

Zusanli (ST36) 3 cun below Yanglingquan (GB34), between the gallbladder and bladder meridian

Liangqiu(ST34) 3 cun below Zhongdu(GB32), between the stomach meridian and the gallbladder meridian

\section{Criteria for discontinuing or modifying allocated interventions $\{11 \mathrm{~b}\}$}

When acupuncture-related serious adverse reactions occur during the experiment or failure to complete the study for any other reasons. We will record the details in the report for the early termination of the study.

\section{Strategies to improve adherence to interventions $\{11 \mathrm{c}\}$}

At the beginning of the trial, participants will be informed of the experiment procedure again. They can report their personal feelings at any time during the study, and we will give corresponding feedback as appropriate to increase the interactivity and then improve adherence to interventions.

\section{Relevant concomitant care permitted or prohibited during the trial $\{11 d\}$}

Patients cannot eat 8 hours before gastroscopy to ensure a fasting state.

\section{Provisions for post-trial care $\{30\}$}

At the end of the study, we will provide certain amount of subsidy to each subject.

\section{Outcomes $\{12\}$}

\section{Primary outcome}

A visual analogue scale (VAS) will be used to evaluate the patient's level of discomfort, which is a line segment from 0 to 10, with 0 indicating low and 10 high discomfort. All patients will complete 4 detailed VAS at 30 minutes and 7 days post-procedure. The questionnaire will evaluate 4 areas of discomfort including nausea and vomiting, throat discomfort, bucking and agitation. The primary outcome is the average score of the 4 VAS scales

\section{Secondary outcomes}


- Cardiac-respiratory assessment:

All patients' oxygen saturation (Sp02), heart rate (HR) and blood pressure (BP). measurements will be recorded prior to, during and post-procedure. Then, each patient's 'double product' (DP) will be calculated at each point of the procedure, based on the systolic blood pressure (SBP) and heart rate (SBP囚HR). DP is a useful prognostic tool for patients with poor cardiac function during exercise and rest, reflecting myocardial oxygen consumption and cardiac output during treadmill testing. A value of DP greater than 15,000 indicates cardiovascular stress. It is assumed that changes in cardiac output from baseline (preoperative) will reflect the level of stress experienced by patients undergoing the gastroscopy.

- Anxiety level:

A six item short form of the State-Trait Anxiety Inventory (STAI-S6) and Amsterdam Pre-operative Anxiety and Information Scale (APAIS) will assess the degree of preoperative anxiety, postoperative anxiety and 7 days post-procedure. These questionnaires are quick and simple for participants to complete, which is advantageous in time-restricted studies.

Preference in a future endoscopy:

The questionnaire will ask patients whether they have undergone gastroscopy before, and then ask, "There are two options to choose when accept next gastroscopy, accept acupuncture before gastroscopy or do not accept acupuncture before gastroscopy."

- Safety assessment

Before participants sign the informed consent form, they will be informed that acupuncture clinical trials may have potential adverse events such as bleeding, hematomas, fainting, bruising, ecchymoma and possible infection. All details of the adverse event will be recorded by the patients and doctors. Adverse events (AE) are rated as 1 (mild), 2 (moderate) or 3 (severe or medically significant). All details of AEs will be recorded in the Case Report Form (CRF). At the end of the trial, we will analyze the impact of all events.

- Blinding success assessment

After the gastroscopy procedure researchers will evaluate the success of the blinding by asking participants the following question: "what kind of treatment do you think you have received?" The options are: traditional acupuncture treatment; acupuncture-like simulation treatment; and uncertain. If participants do not choose "uncertain," then the researcher will question the patient further for specific reasons.

\section{Participant timeline $\{13\}$}

The timing of intervention and data collection is detailed in Fig. 2. 


\section{Sample size $\{14\}$}

In our pilot study, the discomfort VAS assessment at 30 minutes post-procedure was 4.8 (SD, 1.76) in the EA group and 6.3 (SD, 1.53) in the SEA group. We used PASS software to calculate that 50 patients would be needed to provide $90 \%$ power to detect a difference of discomfort VAS in 2 groups at a 2-sided significance level of $5 \%$. If we assume that $20 \%$ of patients would be lost to follow-up, 60 would need to be enrolled.

\section{Recruitment $\{15\}$}

Participants will be recruited through hospital-based advertisements within the outpatient clinic and on the Shanghai Municipal Hospital of TCM website. Research assistants will screen the patients, obtain written informed consent, and assign them to either the EA or SEA group, receiving a traditional acupuncture or an acupuncture-like simulation treatment.

\section{Assignment of interventions: allocation Sequence generation $\{16 \mathrm{a}\}$}

In this trial, we plan to use SPSS24.0 software to generate a random number table, which divides eligible participants into the EA group or SEA group with a 1:1 ratio.

\section{Concealment mechanism $\{16 \mathrm{~b}\}$}

A random distribution card will be made and sealed with an opaque envelope. Participants will be informed that they have an equal chance of being assigned to the EA or SEA group before the trial.

\section{Implementation \{16c\}}

An independent researcher that blinded to the study protocol generate the allocation sequence. Another researcher will give the opaque envelope to acupuncturist according to the timing sequence of participant registration for the trial.Then, acupuncturist open the envelope to perform corresponding acupuncture operation.

\section{Assignment of interventions: Blinding Who will be blinded $\{17 \mathrm{a}\}$}

This is a single-blinded (patient-assessor-blinded) study. To ensure that participants are blind, they will be treated in an isolated space and be required to wear blindfolds. Only the acupuncturist who performs the treatment will know the group assignment at the time of treatment. Gastroenterologists, data analysts and statisticians will remain blinded. 


\section{Procedure for unblinding if needed $\{17 \mathrm{~b}\}$}

Unmasking is not needed.

\section{Data collection and management}

\section{Plans for assessment and collection of outcomes $\{18 \mathrm{a}\}$}

We will timely record the evaluation results in the CRFs.

Plans to promote participant retention and complete followup $\{18 b\}$

Patients came to the hospital one week after gastroscopy to get the result of pathological organization, We followed up at this time point.At the end, gave a certain transportation subsidy.

\section{Data management $\{19\}$}

Patient characteristics will be recorded in the CRFs and stored in the researcher's office. The clinical trial management platform ResMan will be used to manage the raw data. The raw data will be collected by assessors who are blinded to the group assignment and repeated input methods will be used to ensure that the entered data is correct. Before the platform is officially launched, it will be tested and the users will be trained. After the platform is officially launched, the database will be locked with a password, which only be known by relevant personnel. The clinical director will oversee the work of the clinical trial center at least once a month.

\section{Confidentiality $\{27\}$}

All the documents and materials related to this will be kept strictly confidential. Only when principal Investigator allowed will the documents promulgated to the third parties. We protect the privacy of participants' personal medical information within the sphere permitted by law. The staff in this study is also bound by this agreement.

\section{Plans for collection, laboratory evaluation and storage of biological specimens for genetic or molecular analysis in this trial/future use $\{33\}$}

Not applicable, this trial does not have biological specimens.

\section{Statistical methods}




\section{Statistical methods for primary and secondary outcomes $\{20$ a $\}$}

The independent statistician is responsible for the statistical analysis with spss24.0 software. All data will be analyzed in terms of intention to treat (ITT) principle, including data from participants who withdraw during the trial. In the statistical analysis, measurement data between the two groups will be analyzed with the t-test, and rank- sum test, while categorical data will be analyzed with $\chi 2$ test. Data will be recorded as mean \pm standard deviation or median (first quartile, third quartile). The significance level used for statistical analysis will be two-sided with confidence intervals at the $95 \%$ level.

\section{Interim analyses $\{21 \mathrm{~b}\}$}

Not applicable.

\section{Methods for additional analyses (e.g. subgroup analyses) $\{20 b\}$}

Not applicable.

\section{Methods in analysis to handle protocol non-adherence and any statistical methods to handle missing data $\{20 \mathrm{c}\}$}

We will adopt ITT principle to reduce deviation. The missing data will be substituted into the subjects' baseline data for final data analysis.

\section{Plans to give access to the full protocol, participant level- data and statistical code $\{31 \mathrm{c}\}$}

The data sharing will be conducted in accordance with the regulatory requirements.

\section{Oversight and monitoring}

\section{Composition of the coordinating centre and trial steering committee $\{5 \mathrm{~d}\}$}

In order to control the quality of the clinical trial, the whole process of the trial will be conducted under the supervision of a qualified clinical trial expert in Shanghai Municipal Hospital of Traditional Chinese Medicine. The Clinical Research Center for Drugs of Shanghai University of Traditional Chinese Medicine will conduct the data monitoring. When problems occur in the trial, the center has the right to make the final decision to terminate the trial if necessary. Any changes will be notified in writing to all participants 
in the trial after approval by the ethics committee. The principle investigator will be fully responsible for conducting the trial and will make any final decisions.

\section{Adverse event reporting and harms $\{22\}$}

We will record the adverse events such as bleeding, hematomas, fainting, bruising, ecchymoma and possible infection, and analyzed.

\section{Frequency and plans for auditing trial conduct $\{23\}$}

We will audit every three months,any modification that may have an impact on the study, potential benefit to patients, or affect patient safety, including changes of the study objective, study design, patient population, sample size, study procedure or serious adverse events will be reported to the Committee. This will be decided jointly with the monitoring Committee, the Clinical Research Center of Drugs and approved by the Ethics Committee.

\section{Dissemination plans \{31a\}}

The publication of the outcomes of this study will provide baseline data. The outcomes may be presented at conferences, symposiums, teaching classes, etc., if applicable.

\section{Discussion}

Acupuncture therapy has been used in clinical practice on relieving symptoms caused by gastroscopy. For example, Z N Kain ${ }^{14}$ shows that acupuncture may reduce the side effect of vomiting during gastroscopy through the ways of adjusting the function of vegetative nerves, regulating gastrointestinal tract function, reducing the concentration of blood 5-HT. Taras I $\mathrm{U}^{15}$ found that auricular acupuncture $(A A)$ is a promising alternative treatment for situational anxiety, Andrzejowski $\mathrm{JC}^{16}$ found that acupuncture at the EX-HN3 point reduces pre-operative anxiety levels in patients awaiting neurosurgery. However, the existing literature regarding those practices are largely empirical and with inevitable deficits such as a small sample size. Some papers have reported that acupuncture when applied before gastroscopy has no apparent differences compared to sham acupuncture. ${ }^{17-19}$ So, in this trial, we used the study by Liu et $\mathrm{al}^{20}$, which has been widely used in clinical practice as a successful implementation of sham acupuncture, to rule out the placebo effect of acupuncture in a rigorous way. Studies have shown that the superficial acupuncture in the non-acupoints method has the advantages of good masking effect, not easily perceived by the patients, and with higher feasibility of operation. When the clinical trial of acupuncture treatment for pain was carried out, superficial acupuncture in the nonacupoints method is frequently used as the placebo acupuncture way. ${ }^{21}$ By designing this RCT, we aim to contribute better evidence for the effectiveness of electroacupuncture in reducing discomfort during gastroscopy through an improved design and methods.

For patients, surgical procedures performed in hospitals are a strong stressor and can lead to an anxiety response. Excessive anxiety can affect immune mechanisms through the release of corticosteroids and 
may be associated with abnormal hemodynamics as a consequence of endocrine changes and activation of the central nervous system. ${ }^{22,}{ }^{23} \mathrm{~A}$ high level of preoperative anxiety will also reduce patients' satisfaction with the procedure and increase the risk of postoperative nausea and vomiting. ${ }^{24,25}$ So, in this trial, we will focus on preoperative anxiety. The STAI-S6 and APAIS are reliable means of assessing patient anxiety. ${ }^{26,27}$ By using these questionnaires, we can quickly and accurately assess patients' anxiety level in a few minutes.

Previous findings have shown that gastroscopy may bring a series of complications to patients with hypertension, such as myocardial infarction, cardiac arrest and other complications. ${ }^{28}$ Some research has shown that in patients older than 45 years, the risk of cardiovascular disease doubles with an increase in systolic blood pressure of $20 \mathrm{mmHg}$ or a diastolic blood pressure increases of $10 \mathrm{mmHg}{ }^{29,30}$ So, in our study, we will monitor BP before, during and after gastroscopy, then analyze the correlation between DP and the primary outcome of VAS score to determine whether EA can reduce stress placed on the heart, and thus benefit elderly people with cardiovascular related diseases who need gastroscopy.

There are several limitations in this study. First of all, we did not limit any comorbidities that patients may have. Patients who meet our inclusion criteria and need upper gastrointestinal endoscopy according to the assessment of a gastroenterologist can be included in this trial. Some comorbidities like pharyngitis might increase sensitivity and discomfort when the gastroscope passes through the pharynx. Therefore, we cannot precisely determine the impact of comorbid diseases on the level of anxiety during gastroscopy. Secondly, patients will receive a local anesthetic orally after acupuncture, and then wait for several minutes to carry out endoscopic examination. The function of this drug is to make local anesthesia of the throat, eliminating the foam to make the vision of the gastroscope clearer. It cannot be ruled out that the use of this anesthetic may affect the patient's judgment of the questionnaire. Finally, it is inevitable for the acupuncturist to know the grouping of patients. We hope that this limitation will be offset by the fact that the acupuncturist does not participate in the evaluation of the questionnaire and that the patients are subjected to rigorous blinding practices.

We aim to design a standard and rigorous RCT to reduce discomfort during gastroscopy by acupuncture treatment. We believe acupuncture will increase patient compliance and ease burden on public health services. With positive results, our research will serve as a new standard of care for patients hoping to forego systemic sedation during gastroscopy procedures.

\section{Trial Status}

We started recruiting participants in February 2021 and the recruitment is expected to end late 2021.

\section{Abbreviations}

VAS: Standard Visual Analogue Scales 
STAI-S6: The 6 Item Short Form of the State-Trait Anxiety Inventory

APAIS: Amsterdam Pre-Operative Anxiety and Information Scale

Sp02: oxygen saturation

HR: heart rate

BP: blood pressure

STRICTA: Standards for Reporting Interventions in Clinical Trials of Acupuncture

\section{Declarations}

\section{Acknowledgements}

We would like to thank Dr. Lao for guiding experimental design. Shifen Xu provided general support as the head of the Acupuncture Department in Shanghai Municipal Hospital of Traditional Chinese Medicine, and was responsible for the design of this RCT. Thanks for Gastroscope Center of Shanghai Municipal Hospital of Traditional Chinese Medicine.

\section{Authors' contributions $\{31 \mathrm{~b}\}$}

Shifen Xu and Binyu Yu conceived the design of the trial and Lixing Lao revised it. Philippa Jemma Hazlewood and Xuan Yin participated in the design of the statistical analysis. Shanshan Li, Hongyu Yue, Kun Xu, and Yiqun Mi helped in the recruitment of patients. Binyu Yu, Philippa Jemma Hazlewood, and Xuan Yin helped in drafting the paper and quality control. All authors read and approved the final draft of the paper.

\section{Funding $\{4\}$}

This research is supported by the Shanghai Hospital Development Center (grant SHDC2020CR3015A).

\section{Availability of data and materials $\{29\}$}

All data generated or analyzed during this study are included in this published article and its supplementary information files.

\section{Ethics approval and consent to participate $\{24\}$}

This trial has been approved by the Ethics Committee of Shanghai Municipal

Hospital of Traditional Chinese Medicine (2020SHL-KY-11) with the Helsinki

Declaration. Each participant will be clearly aware of the purpose, procedure, and the potential risk of this trial before enrollment. Then give us their written informed consent. 


\section{Consent for publication $\{32\}$}

Not applicable

\section{Competing interests $\{28\}$}

The authors declare that they have no competing interests.

\section{References}

1. Januszewicz W, Kaminski MF. Quality indicators in diagnostic upper gastrointestinal endoscopy. Therap Adv Gastroenterol. 2020;13:1756284820916693.

2. Nishizawa T, Suzuki H. Propofol for gastrointestinal endoscopy. United European Gastroenterol J. 2018;6(6):801-05.

3. Zhang XL, Lu ZS, Tang P, Kong JY, Yang YS. Current application situation of gastrointestinal endoscopy in China. World J Gastroenterol. 2013;19(19):2950-5.

4. Wu L, Zhao H, Weng H, Ma D. Lasting effects of general anesthetics on the brain in the young and elderly: "mixed picture" of neurotoxicity, neuroprotection and cognitive impairment. J Anesth. 2019;33(2):321-35.

5. Zheng HR, Zhang XQ, Li LZ, Wang YL, Wei Y, Chen YM, et al. Multicentre prospective cohort study evaluating gastroscopy without sedation in China. $\mathrm{Br} J$ Anaesth. 2018;121(2):508-11.

6. Wang HL, Ye F, Liao WF, Xia B, Zheng GR. Unsedated versus sedated gastrointestinal endoscopy: a questionnaire investigation in Wuhan, central China. J Huazhong Univ Sci Technolog Med Sci. 2013;33(6):857-61.

7. Yuan W, Wang Q. Perioperative acupuncture medicine: a novel concept instead of acupuncture anesthesia. Chin Med J (Engl). 2019;132(6):707-15.

8. Acar HV. Acupuncture and related techniques during perioperative period: A literature review. Complement Ther Med. 2016;29:48-55.

9. Yang J, Jiang Y, Chen Y, Sun M, Chen J, Zheng Q, et al. Acupressure the PC6 point for alleviating postoperative nausea and vomiting: A systematic review protocol. Med (Baltim). 2019;98(33):e16857.

10. Wu MS, Chen KH, Chen IF, Huang SK, Tzeng PC, Yeh ML, et al. The Efficacy of Acupuncture in PostOperative Pain Management: A Systematic Review and Meta-Analysis. PLoS One. 2016;11(3):e0150367.

11. Sodipo JO, Ogunbiyi TA. Acupuncture analgesia for upper gastrointestinal endoscopy: a Lagos experience. Am J Chin Med. 1981;9(2):171-3.

12. Moher D, Hopewell S, Schulz KF, Montori V, Gotzsche PC, Devereaux PJ, et al. CONSORT 2010 explanation and elaboration: updated guidelines for reporting parallel group randomised trials. Int $\mathrm{J}$ Surg. 2012;10(1):28-55. 
13. Wen W, Yang L, Liu S, Zhong Y, Hu X, Huang X, et al. [Reporting quality and effect size comparison in randomized controlled trials of bo's abdominal acupuncture using CONSORT statement and STRICTA]. J Tradit Chin Med. 2016;36(3):382-91.

14. Wang SM, Peloquin C, Kain ZN. The use of auricular acupuncture to reduce preoperative anxiety. Anesth Analg. 2001;93(5):1178-80. table of contents.

15. Wunsch JK, Klausenitz C, Janner H, Hesse T, Mustea A, Hahnenkamp K, et al. Auricular acupuncture for treatment of preoperative anxiety in patients scheduled for ambulatory gynaecological surgery: a prospective controlled investigation with a non-randomised arm. Acupunct Med. 2018;36(4):222-27.

16. Wiles MD, Mamdani J, Pullman M, Andrzejowski JC. A randomised controlled trial examining the effect of acupuncture at the EX-HN3 (Yintang) point on pre-operative anxiety levels in neurosurgical patients. Anaesthesia. 2017;72(3):335-42.

17. Melchart D, Streng A, Hoppe A, Brinkhaus B, Witt C, Wagenpfeil S, et al. Acupuncture in patients with tension-type headache: randomised controlled trial. BMJ. 2005;331(7513):376-82.

18. Witt C, Brinkhaus B, Jena S, Linde K, Streng A, Wagenpfeil S, et al. Acupuncture in patients with osteoarthritis of the knee: a randomised trial. Lancet. 2005;366(9480):136-43.

19. Diener HC, Kronfeld K, Boewing G, Lungenhausen M, Maier C, Molsberger A, et al. Efficacy of acupuncture for the prophylaxis of migraine: a multicentre randomised controlled clinical trial. Lancet Neurol. 2006;5(4):310-6.

20. Liu Z, Liu J, Zhao Y, Cai Y, He L, Xu H, et al. The efficacy and safety study of electro-acupuncture for severe chronic functional constipation: study protocol for a multicenter, randomized, controlled trial. Trials. 2013;14:176.

21. Mao WC, Liu BY, He LY, Liu ZS, Liu J, Zhao Y. [Discussion on existing problems of placebo acupuncture design based on acupuncture analgesia]. Zhen Ci Yan Jiu. 2013;38(2):163-7.

22. Markland D, Hardy L. Anxiety, relaxation and anaesthesia for day-case surgery. Br J Clin Psychol. 1993;32(4):493-504.

23. Basak F, Hasbahceci M, Guner S, Sisik A, Acar A, Yucel M, et al. Prediction of anxiety and depression in general surgery inpatients: A prospective cohort study of 200 consecutive patients. Int J Surg. 2015;23(Pt A):18-22.

24. Acar HV, Cuvas O, Ceyhan A, Dikmen B. Acupuncture on Yintang point decreases preoperative anxiety. J Altern Complement Med. 2013;19(5):420-4.

25. Bae H, Bae H, Min BI, Cho S. Efficacy of acupuncture in reducing preoperative anxiety: a metaanalysis. Evid Based Complement Alternat Med. 2014;2014:850367.

26. Moerman N, van Dam FS, Muller MJ, Oosting H. The Amsterdam Preoperative Anxiety and Information Scale (APAIS). Anesth Analg. 1996;82(3):445-51.

27. Mohd Fahmi Z, Lai LL, Loh PS. Validation of the Malay version of the Amsterdam Preoperative Anxiety and Information Scale (APAIS). Med J Malaysia. 2015;70(4):243-8. 
28. Murray AW, Morran CG, Kenny GN, Macfarlane P, Anderson JR. Examination of cardiorespiratory changes during upper gastrointestinal endoscopy. Comparison of monitoring of arterial oxygen saturation, arterial pressure and the electrocardiogram. Anaesthesia. 1991;46(3):181-4.

29. Amornyotin S, Lertakayamanee N, Wongyingsinn M, Pimukmanuskit P, Chalayonnavin V. The effectiveness of intravenous sedation in diagnostic upper gastrointestinal endoscopy. $J$ Med Assoc Thai. 2007;90(2):301-6.

30. Zhao P, Xiao SM, Tang LC, Ding Z, Zhou X, Chen XD. Proximal gastrectomy with jejunal interposition and TGRY anastomosis for proximal gastric cancer. World J Gastroenterol. 2014;20(25):8268-73.

\section{Figures}




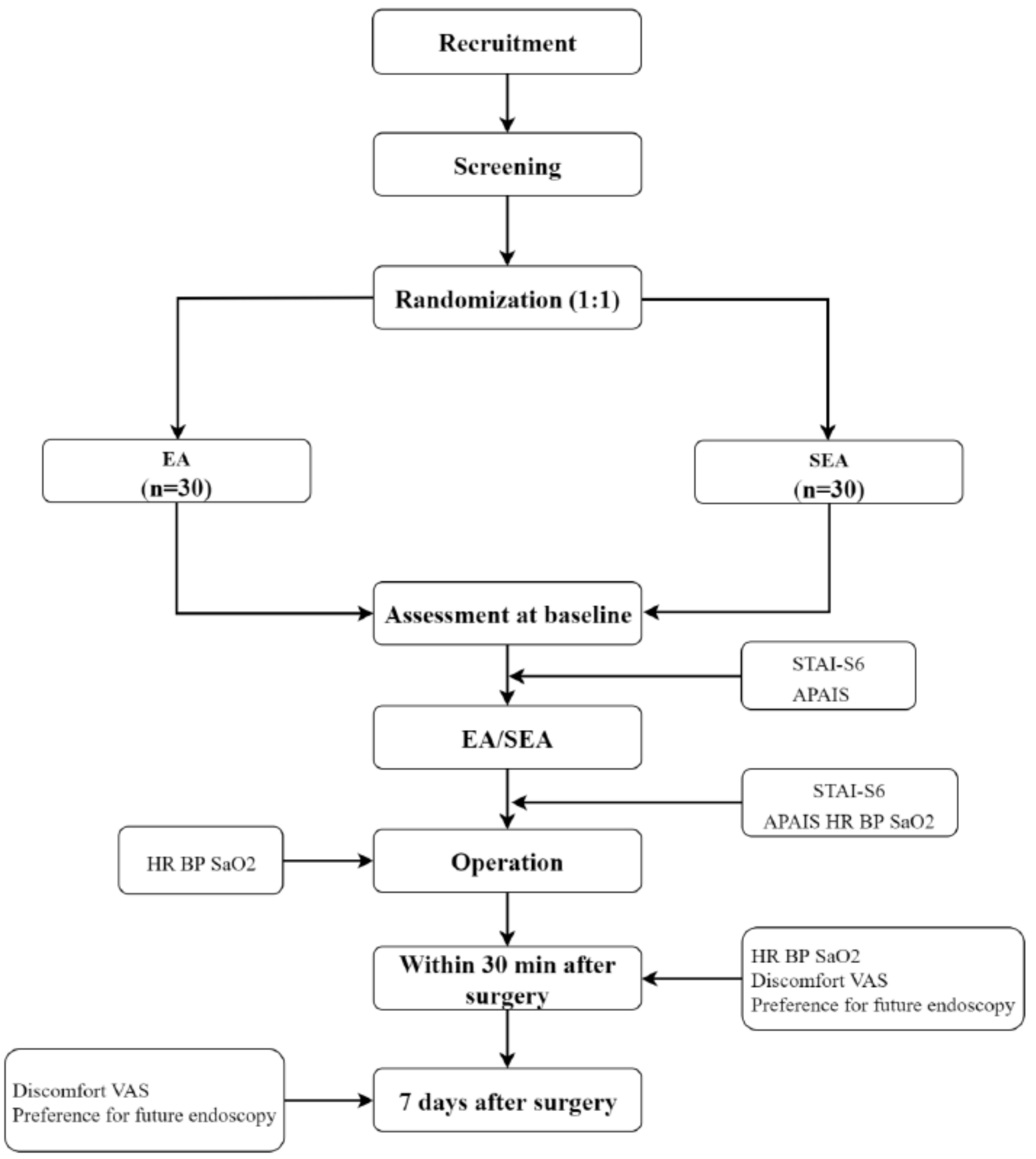

\section{Figure 1}

Flowchart of this study 


\begin{tabular}{|c|c|c|c|c|c|c|c|}
\hline \multirow[t]{2}{*}{ Timepoint } & Screening & Baseline & Intervention & & Operation & & Follow-up \\
\hline & & & before after & Before & During & $\begin{array}{c}30 \\
\text { minutes } \\
\text { after } \\
\text { endoscopy }\end{array}$ & Day 7 \\
\hline
\end{tabular}

Basic

information

$\begin{array}{lll}\text { Informed consent } & X & \\ \text { Inclusion/exclusion } & X & X\end{array}$

Interventions

$\begin{array}{lll}\text { EA } & X & X \\ \text { SEA } & X & X\end{array}$

Assessments

Primary outcome

Discomfort VAS

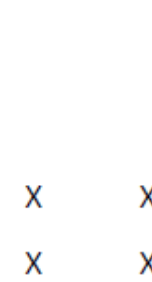

APAIS

HR BP and SpO2

Others

Adverse events

X $\quad \mathrm{x}$

$\mathrm{X}$

$\mathrm{X}$

X

X

Patient satisfaction

Success of blinding
X

X

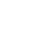

Secondary
outcomes
STAI-S6
APAIS
HR BP and SpO2

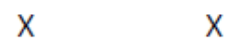

$x$

EA electroacupuncture, SEA sham electroacupuncture, VAS visual analogue scales, STAI-S6 six item short form of the state-trait anxiety inventory, APAIS Amsterdam pre-operative anxiety and information scale, HR heart rate, BP blood pressure. SaO2 oxygen saturation

Figure 2

Schedule of enrolment, intervention, and assessments

\section{Supplementary Files}

This is a list of supplementary files associated with this preprint. Click to download.

- Picture1.jpg

- Picture2.jpg

- Picture3.jpg

- Picture4.jpg 